\title{
Models of longitudinal space-charge impedance for microbunching instability
}

\author{
Marco Venturini* \\ Lawrence Berkeley National Laboratory, University of California, Berkeley, California 94720, USA
}

(Received 20 August 2007; published 25 March 2008)

\begin{abstract}
A 1D model of space-charge impedance, assuming a transversely uniform beam with circular cross section, has been proposed and is being extensively used in the modeling of the microbunching instability of relevance for the beam delivery systems of x-ray free-electron lasers. In this paper we investigate the limitation of the model when applied to studying the effect of shot noise-one of the sources of the microbunching instability. We make comparison with a fully 3D calculation and identify the upper end of the frequency spectrum for applicability of the $1 \mathrm{D}$ model. Relaxation of the assumptions regarding axis symmetry and uniformity of the transverse density is also reviewed.
\end{abstract}

DOI: 10.1103/PhysRevSTAB.11.034401

PACS numbers: 29.27.Bd, 41.60.Ap

\section{INTRODUCTION}

A successful design of the beam delivery systems for $\mathrm{x}$ ray free-electron lasers (FELs) requires control and hence reliable and efficient modeling of the microbunching instability [1]. At present, two distinct approaches are being pursued for modeling: macroparticle simulations [2-4] and direct solution of the Vlasov equation (in its full $[5,6]$ or linearized form $[7,8])$.

Macroparticle simulations in combination with particle in cell techniques allow for accurate determination of the fields for a given charge density but are sensitive to the unphysical fluctuations arising from using a number of macroparticles smaller than the bunch population. Direct methods to solve the full Vlasov equation are attractive in that they are immune from this source of noise but have limitations on their own. Because of the strong dependence of the computational load on the dimensionality of the system, they are most efficient in a reduced phase space. Indeed, solvers implemented so far have been limited to a 2D (longitudinal) phase space [5,6] and are necessarily based on a simplified model of beam dynamics and, in particular, of space-charge effects.

In this paper we discuss the simplified model of space charge proposed in [9] and adopted in [2,5,6]. This model assumes an infinitely long beam in free space with uniform transverse density and circular cross section and yields an on-axis longitudinal electric field in Fourier space $\tilde{E}_{z}(k)=$ $-Z(k) \tilde{I}(k)$, which is exclusively determined by the beam current $I$ and an impedance $Z(k)$ [10].

The model has the pleasant feature that $Z(k)$ can be cast into a handy analytical expression but has two obvious limitations: (i) the reduced dimensionality (1D); (ii) the assumption regarding uniformity and symmetry of the transverse density. Our main goal here is to address (i) with regard to the electric field generated by shot noise, the most fundamental source of density fluctuations seeding the microbunching instability. At high frequency one may

*mventurini@lbl.gov expect that an evaluation of $\tilde{E}_{z}(k)$ based on a $1 \mathrm{D}$ beam model would fail when the wavelength of the charge perturbation (in the beam frame) becomes comparable to the beam transverse size. In Sec. IV we identify this critical wavelength by making a comparison between the expectation values $\left\langle\left|\tilde{E}_{z}(k)\right|^{2}\right\rangle$ as calculated from a 3D and the 1D model. We find that the two quantities start to diverge significantly for $k r_{b} / \gamma \gtrsim 0.5$, where $r_{b}$ is the radius of the beam transverse cross section. This is the regime where the transverse correlation length for $\tilde{E}_{z}(k)$ becomes comparable to, or smaller than, the beam transverse size. (In the above equation the average $\langle\cdot\rangle$ is taken over the random realizations of the charge density due to shot noise.)

As for the remainder of the paper, in Sec. III we review the 1D model of space-charge (SC) impedance for uniform axis-symmetric beam, while in the last two sections we briefly discuss beams with transverse Gaussian densities and non-axis-symmetric profiles, and for completeness we review the well-known effect of conducting walls (which limits the validity of simplified 1D models of space charge in free space from the low-frequency side of the frequency spectrum).

\section{BASIC EQUATIONS FOR THE ELECTRIC FIELD}

Consider an infinitely long electron beam with density $\lambda \rho(x, y, z)$, where $\lambda$ is the (uniform) linear particle density. More specifically, we will consider densities of the form $\rho(x, y, z)=\rho_{\perp}(x, y) \rho_{z}(z)$ with normalization $\int \rho_{\perp}\left(x^{\prime}, y^{\prime}\right) d x^{\prime} d y^{\prime}=1$ and $\int_{-L / 2}^{L / 2} d z \rho_{z}(z)=L$ for $L \rightarrow$ $\infty$. The beam moves in free space with constant velocity $\beta c$ in the $z$ direction (we shall assume $\beta \simeq 1$ ). In the lab frame the longitudinal electric field generated by such a beam is given by $E_{z}(\boldsymbol{x})=\left(e \lambda / 4 \pi \varepsilon_{0}\right) \int G\left(\boldsymbol{x}, \boldsymbol{x}^{\prime}\right) \rho\left(\boldsymbol{x}^{\prime}\right) d^{3} \boldsymbol{x}^{\prime}$ with Green function $G\left(\boldsymbol{x}, \boldsymbol{x}^{\prime}\right)=\left(z-z^{\prime}\right) \gamma /\left[\left(x-x^{\prime}\right)^{2}+\right.$ $\left.\left(y-y^{\prime}\right)^{2}+\left(z-z^{\prime}\right)^{2} \gamma^{2}\right]^{3 / 2}$. When working in cylindrical coordinates it is convenient to make use of the expansion [11] 


$$
\begin{aligned}
G\left(\boldsymbol{x}, \boldsymbol{x}^{\prime}\right)= & -\frac{i}{\pi \gamma^{2}} \sum_{m=-\infty}^{\infty} e^{i m\left(\phi-\phi^{\prime}\right)} \int_{-\infty}^{\infty} d k e^{i k\left(z-z^{\prime}\right)} k \\
& \times I_{m}\left(\frac{k r_{<}}{\gamma}\right) K_{m}\left(\frac{k r_{>}}{\gamma}\right),
\end{aligned}
$$

where following Jackson's notation $r_{<}\left(r_{>}\right)$denotes the smaller (larger) between $r$ and $r^{\prime}$, and $I_{m}$ and $K_{m}$ are the modified Bessel functions. The Fourier component $\tilde{E}_{z}(k)=(2 \pi)^{-1} \int_{-\infty}^{\infty} E_{z}(\boldsymbol{x}) e^{-i k z} d z$ of wave number $k$ then reads

$$
\begin{aligned}
\tilde{E}_{z}(k)= & -i \frac{e}{4 \pi \varepsilon_{0}} \frac{\lambda}{\pi \gamma^{2}} \sum_{m=-\infty}^{\infty} \int d V^{\prime} \rho\left(r^{\prime}, \phi^{\prime}, z^{\prime}\right) k e^{i m\left(\phi-\phi^{\prime}\right)} \\
& \times e^{-i k z^{\prime}} k I_{m}^{<} K_{m}^{>},
\end{aligned}
$$

with obvious meaning of the shorthand notation $I_{m}^{<}$and $K_{m}^{>}$.

\section{1D MODEL OF SHOT NOISE}

Assume a transversely uniform density with circular cross section of radius $r_{b}$ and an observation point located on-axis $(r=0)$. Only the $m=0$ term in (2) contributes, while the radial integration can be carried out explicitly using $\int_{0}^{\xi} x K_{0}(x) d x=\left[1-\xi K_{1}(\xi)\right]$. An impedance (per unit length) $Z(k)$ is defined as

$$
\tilde{E}_{z}(k)=-Z(k) \tilde{I}(k),
$$

where $\tilde{I}(k)$ is the Fourier transform of the current $I(z)=$ $e c \lambda \rho_{z}(z)$, with $\tilde{\rho}_{z}(k)=(2 \pi)^{-1} \int_{-\infty}^{\infty} \rho_{z}(z) e^{-i k z} d z$. We have

$$
Z(k)=i \frac{Z_{0}}{\pi \gamma r_{b}} \frac{1-\xi_{b} K_{1}\left(\xi_{b}\right)}{\xi_{b}},
$$

where $\xi_{b}=k r_{b} / \gamma$. It is interesting to report the limiting form of (4) at high and low frequencies. For $x \rightarrow \infty, K_{1}(x)$ decreases exponentially, and therefore $Z(k \rightarrow \infty)=$ $i Z_{0} / \pi k r_{b}^{2}$. For small $x$ we have $K_{1}(x) \simeq 1 / x+$ $x\left[\log (x / 2)+\gamma_{E}-1 / 2\right] / 2$, where $\gamma_{E} \simeq 0.577$ is the Euler constant, yielding

$$
Z(k \rightarrow 0)=-i \frac{Z_{0}}{4 \pi} \frac{k}{\gamma^{2}}\left[\log \frac{\xi_{b}^{2}}{4}+2 \gamma_{E}-1\right] .
$$

The granularity of the elementary charge gives rise to random fluctuations of the beam current (shot noise). We are interested in investigating how charge density fluctuations translate into electric field fluctuations and determining their statistics.

For convenience of calculation consider a beam with long (i.e. longer than any length scale involved in the problem) but finite length $L$. Consider a subdivision of $L$ into $\mathcal{N}$ intervals of length $\Delta z=L / \mathcal{N}$. Denote with $N_{b}=$ $\lambda L$ the total number of electrons in the beam and $N_{j}$ the population of electrons in the interval $z \in \Delta z[j-1, j]$. The occupation number $N_{j}$ is a random process obeying the Poisson statistics, which we model as [12]

$$
N_{j}=\left\langle N_{j}\right\rangle+\left\langle N_{j}\right\rangle^{1 / 2} \eta_{j},
$$

where the expectation value over noise realizations is $\left\langle N_{j}\right\rangle=\lambda \Delta z=N_{b}(\Delta z / L)$ and $\eta_{j}$ is a univariate normal random process with vanishing average and variance equal to unity $\left\langle\eta_{j}\right\rangle=0,\left\langle\eta_{i} \eta_{j}\right\rangle=\delta_{i j}$. The last equation expresses the assumed lack of correlation between the number of electrons populating different intervals. From $\lambda \rho_{z}\left(z_{j}\right) \Delta z=N_{j}$ it follows

$$
\rho_{z}\left(z_{j}\right)=1+\frac{\eta_{j}}{\left\langle N_{j}\right\rangle^{1 / 2}}
$$

and $\left\langle\rho_{z}\left(z_{i}\right) \rho_{z}\left(z_{j}\right)\right\rangle=1+\delta_{i j} /\left\langle N_{j}\right\rangle$. As for the corresponding Fourier transforms (for $k \neq 0, k^{\prime} \neq 0$ ) we have

$$
\left\langle\tilde{\rho}_{z}(k)\right\rangle=0, \quad\left\langle\tilde{\rho}_{z}(k) \tilde{\rho}_{z}^{*}\left(k^{\prime}\right)\right\rangle=(2 \pi)^{-2}\left(L^{2} / N_{b}\right) \delta_{k, k^{\prime}},
$$

where $*$ denotes complex conjugation. Because $L$ is finite it is understood that only a spectrum of discrete wave numbers is allowed: $k=k_{n}=2 \pi n / L$.

From (3) and (8), $\left\langle\tilde{E}_{k}\right\rangle=0$ follows, and after taking the limit $L \rightarrow \infty$

$$
\begin{aligned}
\left\langle\tilde{E}_{k} \tilde{E}_{k^{\prime}}^{*}\right\rangle & =\frac{(e c)^{2}}{(2 \pi)^{2}} N_{b}|Z(k)|^{2} \delta_{k, k^{\prime}} \\
& =\frac{e^{2}}{\left(2 \pi^{2} \varepsilon_{0} \gamma r_{b}\right)^{2}} N_{b}\left(\frac{1-\xi_{b} K_{1}\left(\xi_{b}\right)}{\xi_{b}}\right)^{2} \delta_{k, k^{\prime}},
\end{aligned}
$$

where for brevity we have introduced the notation $\tilde{E}_{k}=$ $\tilde{E}_{z}(k)$. At low frequency $\xi_{b} \rightarrow 0$, we have the limiting form $\left[1-\xi_{b} K_{1}\left(\xi_{b}\right)\right]^{2} / \xi_{b}^{2} \rightarrow \xi_{b}^{2}\left[\log \left(4 \xi_{b}^{2}\right)-2 \gamma_{E}+1\right]^{2} / 16$.

\section{A 3D MODEL OF SHOT NOISE}

Next, we want to contrast (9) to the result obtained from a 3D model of the shot noise. Again, we consider a beam with circular cross section but with transverse density that is uniform only on average: $\left\langle\rho_{\perp}(r)\right\rangle_{\perp} \equiv \rho_{\perp 0}=1 / \pi r_{b}$ for $r<r_{b}$ (and vanishing for $r>r_{b}$ ). Consider an elementary cell of volume $r_{i} \Delta r \Delta \phi \Delta z$ centered at $r_{i}, \phi_{\ell}, z_{j}$. Denote with $N_{i \ell j}$ the number of electrons populating this volume cell. Similarly to (6) we have

$$
N_{i \ell j}=\left\langle N_{i \ell j}\right\rangle+\left\langle N_{i \ell j}\right\rangle^{1 / 2} \eta_{i} \eta_{\ell} \eta_{j}
$$

with the average number of electrons given by $\left\langle N_{i \ell j}\right\rangle=$ $\lambda \rho_{\perp 0} r_{i} \Delta r \Delta \phi \Delta z$.

To calculate $\left\langle\tilde{E}_{k} \tilde{E}_{k^{\prime}}^{*}\right\rangle$ we first discretize the volume integral in (2), $\int d V^{\prime} \rightarrow \sum_{i \ell_{j}} r_{i} \Delta r \Delta \phi \Delta z$, make use of $\left\langle\rho\left(r_{i}, \phi_{\ell}, z_{j}\right) \rho\left(r_{i^{\prime}}, \phi_{\ell^{\prime}}, z_{j^{\prime}}\right)\right\rangle=1+\delta_{i i^{\prime}} \delta_{j j^{\prime}} \delta_{\ell \ell^{\prime}} /\left\langle N_{i \ell j}\right\rangle$, and take the limit $L \rightarrow \infty$ in the end. We find (for $k \neq 0$ and $k^{\prime} \neq 0$ ) 


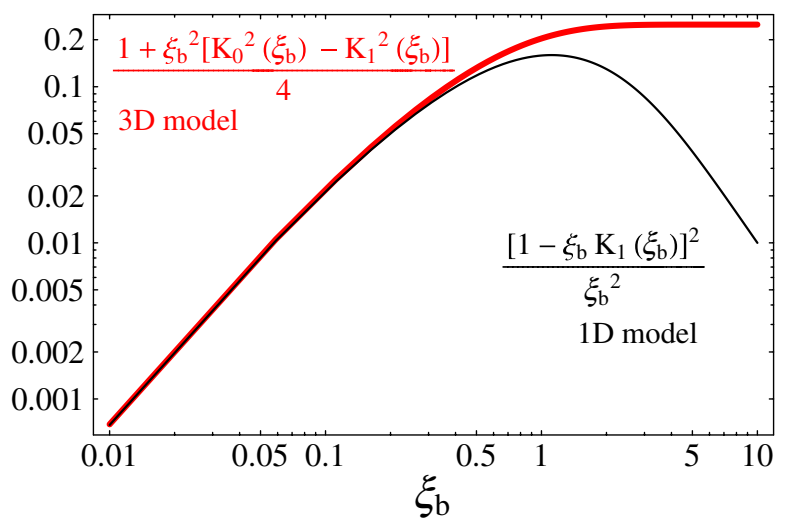

FIG. 1. (Color) The two curves are proportional to the expectation value $\left\langle\left|\tilde{E}_{k}\right|^{2}\right\rangle$ as determined from the 1D model [black curve, Eq. (9)] and 3D model [red curve, Eq. (11)]. The relative difference is less than $10 \%$ up to $\xi_{b} \equiv r_{b} k / \gamma \simeq 0.5$.

$\left\langle\tilde{E}_{k} \tilde{E}_{k^{\prime}}^{*}\right\rangle=\left(\frac{e}{2 \pi^{2} \varepsilon_{0} \gamma r_{b}}\right)^{2} N_{b} \frac{1+\xi_{b}^{2}\left[K_{0}^{2}\left(\xi_{b}\right)-K_{1}^{2}\left(\xi_{b}\right)\right]}{4} \delta_{k, k^{\prime}}$.

For $\quad \xi_{b} \rightarrow 0$, we have $\left[1-\xi_{b} K_{1}\left(\xi_{b}\right)\right]^{2} / \xi_{b}^{2} \rightarrow$ $\xi_{b}^{2}\left\{\left[\log \left(4 \xi_{b}^{2}\right)-2 \gamma_{E}+1\right]^{2} / 16+1 / 16\right\}$.

The relative difference between (9) and (11) vanishes in the zero-frequency limit and becomes significant only for $\xi_{b} \gtrsim 0.5$ (see Fig. 1). In the high-frequency limit (11) tends to a constant, $\left\{1+\xi_{b}^{2}\left[K_{0}^{2}\left(\xi_{b}\right)-K_{1}^{2}\left(\xi_{b}\right)\right]\right\} / 4 \rightarrow 1 / 4$, in contrast to (9), which decreases as $1 / \xi_{b}^{2}$. We conclude that the 1D model gives a good approximation of the field fluctuations for long wavelengths down to $\lambda \simeq 4 \pi r_{b} / \gamma$.

An interesting quantity is the radial correlation $\left\langle\tilde{E}_{k}(r) \tilde{E}_{k}^{*}(0)\right\rangle$. Again, from (2) we have

$$
\begin{aligned}
\left\langle\tilde{E}_{k}(r) \tilde{E}_{k}^{*}(0)\right\rangle= & \frac{e^{2}}{\left(2 \pi^{2} \varepsilon_{0} \gamma r_{b}\right)^{2}} \frac{N_{b}}{2} \\
& \times\left[K_{0}(\xi) \int_{0}^{\xi} x I_{0}(x) K_{0}(x) d x\right. \\
& \left.+I_{0}(\xi) \int_{\xi}^{\xi_{b}} x K_{0}^{2}(x) d x\right]
\end{aligned}
$$

where $\xi=k r / \gamma$, indicating that the radial correlation of the Fourier spectrum components of the longitudinal fields decreases exponentially at high frequencies $\left(\xi_{b} \gg 1\right)$, see Fig. 2 where the quantity $\left\langle\tilde{E}_{k}(r) \tilde{E}_{k}^{*}(0)\right\rangle /\left\langle\left|\tilde{E}_{k}(0)\right|^{2}\right\rangle$ is plotted as a function of $r / r_{b}$ (solid lines).

Equation (12) should be contrasted to the radial correlation from the 1D model (see dashed lines in Fig. 2):

$$
\begin{aligned}
\left\langle\tilde{E}_{k}(r) \tilde{E}_{k}^{*}(0)\right\rangle= & \frac{e^{2}}{\left(2 \pi^{2} \varepsilon_{0} \gamma r_{b}\right)^{2}} N_{b}\left(\frac{1-\xi_{b} K_{1}\left(\xi_{b}\right) I_{0}(\xi)}{\xi_{b}}\right) \\
& \times\left(\frac{1-\xi_{b} K_{1}\left(\xi_{b}\right)}{\xi_{b}}\right) .
\end{aligned}
$$

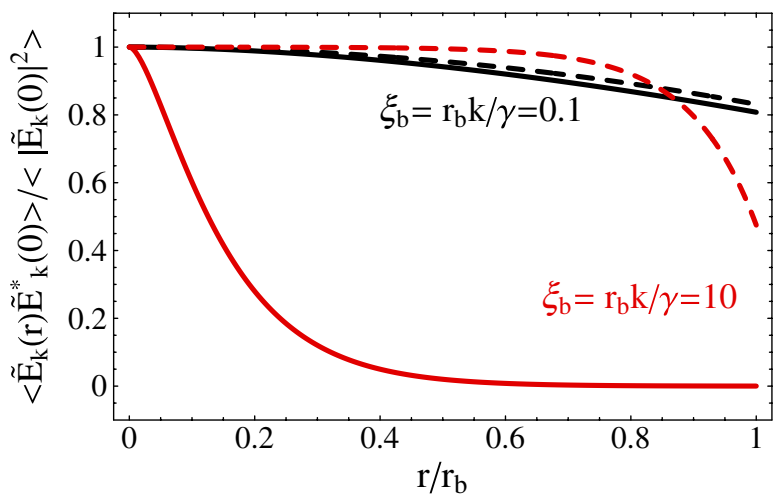

FIG. 2. (Color) At large frequencies $\left(\xi_{b} \gg 1\right)$ the 3D model of shot noise yields an exponentially decreasing radial correlation for the longitudinal electric field (solid red curve), whereas the 1D model gives a strong correlation up to the edge of the beam (dashed red line). At low frequencies $\left(\xi_{b} \ll 1\right)$, the correlations from the 3D (solid black curve) and the 1D model (dashed black curve) are similar and tend to unity.

At low frequencies $\left(\xi_{b} \ll 1\right)$ the correlation (13) tends to (12) and they both tend to unity - a confirmation of the validity of the $1 \mathrm{D}$ model in this regime.

For $\left(\xi_{b} \gg 1\right)$ the limiting form of (12) is well approximated by $\left\langle\tilde{E}_{k}(r) \tilde{E}_{k}^{*}(0)\right\rangle /\left\langle\left|\tilde{E}_{k}(0)\right|^{2}\right\rangle \simeq \xi K_{1}(\xi)$, where the modified Bessel function $K_{1}(\xi) \simeq \sqrt{\pi / 2 \xi} e^{-\xi}$ for $\xi$ of the order of, or larger than, unity. If we define the correlation length $\ell_{c}$ as the radial distance over which the correlation decreases by $1 / e$, we find $\ell_{c} \simeq 1.66 \gamma / k \simeq 0.26 \gamma \lambda$. The $3 \mathrm{D}$ effects start to become important when the correlation $\ell_{c}$ is comparable to or smaller than the beam transverse size $r_{b}$.

The model considered in this section presupposes a frozen beam where interparticle distances do not change significantly in comparison with the correlation length $\ell_{c}$. In contrast, the $1 \mathrm{D}$ model of the previous section only assumes that the longitudinal projection of the interparticle distance does not vary. The time scales over which these assumptions hold are different. Outside dispersive regions of the lattice, the time scale for longitudinal density variations is set by the longitudinal plasma oscillations. In a relativistic regime ( $\gamma \gtrsim 100$ ), say past the injection section in a 4th generation light source, the time scale as measured in terms of the distance traveled by the beam is of the order of $100 \mathrm{~m} \mathrm{[2].} \mathrm{On} \mathrm{the} \mathrm{other} \mathrm{hand,} \mathrm{the} \mathrm{3D} \mathrm{model} \mathrm{is} \mathrm{also}$ sensitive to thermal motion in the transverse plane. One can expect that this may affect transverse correlations of length $\ell_{c}$ over a time of the order $\ell_{c} / v_{\perp}$. [or equivalently over a distance $s_{c} \simeq c\left(\ell_{c} / v_{\perp}\right)$ ], where $v_{\perp}$ is the magnitude of the particles transverse velocity. For example, assuming that $\ell_{c}$ be a fraction of the transverse beam size, say $\ell_{c}=$ $r_{b} / 5$, with $r_{b} \simeq \sqrt{\varepsilon \beta_{x} / \gamma}$ and $v_{\perp} \simeq c \sqrt{\varepsilon / \gamma \beta_{x}}$, we find $s_{c} \simeq$ $\beta_{x} / 5$. For typical values of the betatron function $\beta_{x}, s_{c}$ would be at most of the order of a few meters. This relatively short distance, however, could be sufficient in certain circumstances to induce detectable 3D effects. 


\section{BEAMS WITH NONUNIFORM TRANSVERSE DENSITY}

For an axis-symmetric beam with Gaussian transverse density given by $\rho_{\perp}(x, y)=e^{-\left(x^{2}+y^{2}\right) / 2 \sigma^{2}} / 2 \pi \sigma^{2}$, the onaxis Fourier component of the longitudinal electric field of wave number $k$ reads

$$
\tilde{E}_{k}=i \frac{e}{2 \pi \varepsilon} \frac{\lambda}{\sigma \gamma} \xi_{\sigma} e^{\xi_{\sigma}^{2} / 2} \operatorname{Ei}\left(-\xi_{\sigma}^{2} / 2\right),
$$

where $\xi_{\sigma}=k \sigma / \gamma$ and $\operatorname{Ei}(x)=-\int_{-x}^{\infty} d t e^{-t} / t$ is the exponential-integral function, yielding the impedance

$$
Z(k)=-i \frac{Z_{0}}{\pi \gamma \sigma} \frac{\xi_{\sigma}}{4} e^{\xi_{\sigma}^{2} / 2} \operatorname{Ei}\left(-\frac{\xi_{\sigma}^{2}}{2}\right) .
$$

In deriving (14) we made use of the result [13] $\int_{0}^{\infty} x K_{0}(x) e^{-x^{2} / 2 \xi^{2}} d x=-\left(\xi^{2} / 2\right) e^{\xi^{2} / 2} \operatorname{Ei}\left(-\xi^{2} / 2\right)$.

At large frequencies $Z(k \rightarrow \infty)=i Z_{0} / 2 \pi \sigma^{2} k$ [having used the asymptotic expansion $\operatorname{Ei}(-x)=e^{-x}\left(x^{-1}+\ldots\right)$, valid for large $x$ ]. In the low-frequency limit, since $\operatorname{Ei}(-x) \simeq \gamma_{E}+\log (x)$ for small $x$, we find

$$
Z(k \rightarrow 0)=-i \frac{Z_{0}}{4 \pi} \frac{k}{\gamma^{2}}\left(\log \frac{\xi_{\sigma}^{2}}{2}+\gamma_{E}\right),
$$

which gives a good approximation for $Z(k)$ up to $\xi_{\sigma} \simeq 0.5$. By comparing (5) and (16), one can define an "equivalent" uniform beam with radius $r_{b}=\sigma \sqrt{2} e^{\left(1-\gamma_{E}\right) / 2} \simeq 1.747 \sigma$ to represent the longitudinal impedance for a Gaussian beam with rms size $\sigma$, see Fig. 3 .

If the Gaussian beam has unequal horizontal and vertical rms sizes, similar considerations show that the on-axis 1D space-charge impedance can be approximated with that of a round uniform beam [14] with $r_{b} \simeq 1.747\left(\sigma_{x}+\sigma_{y}\right) / 2$, which is close to the prescription $r_{b}=1.7\left(\sigma_{x}+\sigma_{y}\right) / 2$ proposed in [2] on the basis of numerical fitting.

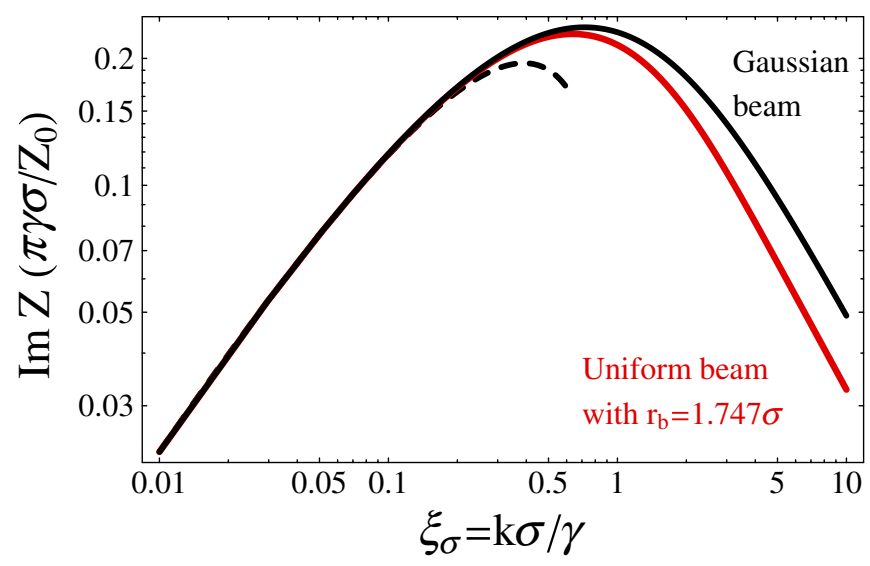

FIG. 3. (Color) The longitudinal (on-axis) impedance for an axis-symmetric Gaussian beam with rms size $\sigma$ (solid black curve) at low frequencies is very close to that of a uniform beam of radius $r_{b}=1.747 \sigma$ (red curve). The dashed curve is the lowfrequency limiting form (16).

\section{EFFECT OF BOUNDARIES}

To estimate the effect of boundaries consider again a model of beam with transverse uniform density and round cross section. In the presence of a perfectly conducting pipe of radius $r_{p}$ concentric with the charge distribution, one can easily show that the impedance reads

$$
\begin{aligned}
Z(k)= & i \frac{Z_{0}}{\pi \gamma r_{b}} \frac{1}{\xi_{b}}\left[1-\xi_{b}\left(K_{1}\left(\xi_{b}\right)+K_{0}\left(\xi_{b} r_{p} / r_{b}\right)\right.\right. \\
& \left.\left.\times \frac{I_{1}\left(\xi_{b}\right)}{I_{0}\left(\xi_{b} r_{p} / r_{b}\right)}\right)\right] .
\end{aligned}
$$

The high-frequency limit $k \rightarrow \infty$ is the same as for the free-space case since both $K_{0}\left(\xi_{b} r_{p} / r_{b}\right)$ and the ratio $I_{1}\left(\xi_{b}\right) / I_{0}\left(\xi_{b} r_{p} / r_{b}\right)$ decrease exponentially with $\xi_{b}$ (as $\left.r_{p} / r_{b}>1\right)$. The low-frequency limit yields the more familiar expression [15]

$$
Z(k \rightarrow 0)=i \frac{Z_{0}}{2 \pi} \frac{k}{\gamma^{2}}\left[2 \log \left(\frac{r_{p}}{r_{b}}\right)+1\right],
$$

which can be recovered from (17) using the limiting expressions $K_{0}(x) \simeq-\left[\gamma_{E}+\log (x / 2)\right], \quad I_{1}(x) \simeq x / 2$, and $I_{0}(x) \simeq 1$, in addition to that for $K_{1}(x)$ reported in Sec. III, for $x \rightarrow 0$. The effect of the boundary becomes significant at low frequencies below $\xi_{b} \simeq r_{b} / r_{p}$ or wavelengths $\lambda \gtrsim 2 \pi r_{p} / \gamma$. For typical pipe apertures this latter quantity is generally larger than the scale of the wavelength of interest for microbunching.

\section{CONCLUSIONS}

We have shown that charge density fluctuations due to shot noise in the transverse plane of the beam can translate into significant fluctuations of the longitudinal electric field with wavelengths smaller than $2 \pi r_{b} / \gamma$ (measured in the laboratory frame). This effect is missed by a purely 1D model of impedance, which yields a longitudinal electric field that responds exclusively to variations in the longitudinal line density. In this paper we have quantified these fluctuations, using a beam model with uniform averaged transverse density and circular cross section, for which a calculation can be carried out analytically. We found that the on-axis expectation value for $\left|\tilde{E}_{k}\right|^{2}$ generated by shot noise tends to a constant at large wave number $k$. In contrast, in the same limit the $1 \mathrm{D}$ model predicts a power-law decay as $1 / k^{2}$. At low frequencies $\left|\tilde{E}_{k}\right|^{2}$ is largely correlated in the transverse plane while at small wavelengths the correlation length $\ell_{c} \simeq 0.26 \gamma \lambda$ can become a small fraction of the transverse beam size. The divergence in the behavior between the 3D and 1D models sets a frequency range delimited by $k r_{b} / \gamma \leq 0.5$ for the validity of the $1 \mathrm{D}$ model.

On the low end of the frequency spectrum where the 1D model is accurate, we showed that the impedance derived for a beam with transversely uniform density and circular 
cross section of radius $r_{b}$ can be used to reproduce with good accuracy the on-axis longitudinal field of a Gaussian beam provided that the parameter $r_{b}$ be adjusted appropriately.

We end with a few words about the practical implications of these findings.

In applications to 4 th generation light sources, beam density fluctuations of very small length scale may be ignored if the smoothing effect of finite uncorrelated energy spread and horizontal emittance are significant. If this is the case, the frequency upper limit we have identified may not pose a particularly restrictive limitation to the applicability of the 1D model of space charge for microbunching.

For example, for FERMI [16] right after injection where $\gamma \simeq 200$ and $r_{b} \simeq 200 \mu \mathrm{m}$, the 1D model is valid for wavelengths longer than $\lambda=4 \pi r_{b} / \gamma \simeq 12 \mu \mathrm{m}$.

This is below the wavelength range of the microbunching gain function through the bunch compressors, which is peaked at about $\lambda=60 \mu \mathrm{m}$ and negligible below $\lambda=$ $20 \mu \mathrm{m}$ [6], provided that the relative uncorrelated energy spread, as resulting from use of a laser heater, is not smaller than about $10^{-4}$. However, turning off the laser heater [17] would move the range of significant microbunching gain to a region well below $\lambda=20 \mu \mathrm{m}$, where the applicability of the 1D model could become questionable. The validity of the 1D model is also in doubt at the end of the FERMI linac where the relative uncorrelated energy spread is small (because of the large beam energy) and microbunching on the $1 \mu \mathrm{m}$ scale could develop in the spreader [18] region. (For a discussion see [19].) Finally, we should mention that evidence of microbunching in mediumenergy, longitudinally cold beams that appears to be inconsistent with a simple 1D model of longitudinal space charge has been gathered in recent measurements of coherent transition radiation signals at Linac Coherent Light Source (LCLS) [20]. These measurements point to the presence of correlations in the beam transverse plane with correlation lengths smaller than the beam transverse size [21].

\section{ACKNOWLEDGMENTS}

I would like to thank J. Qiang and A. Zholents for useful discussions and comments. I would also like to thank D. H. Dowell, A. Chao, Z. Huang, and D. Ratner for sharing early measurements from the LCLS injector commissioning and insight on the interpretation of those measurements. This work was carried out under Department of
Energy Contract No. DE-AC02-0SCK11231.

[1] E. L. Saldin, E. A. Schneidmiller, and M. V. Yurkov, Nucl. Instrum. Methods Phys. Res., Sect. A 483, 516 (2002).

[2] Z. Huang, M. Borland, P. Emma, J. Wu, C. Limborg, G. Stupakov, and J. Welch, Phys. Rev. ST Accel. Beams 7, 074401 (2004).

[3] I. Pogorelov et al., Proceedings of PAC07, Albuquerque, New Mexico, USA (IEEE, Piscataway, NJ, 2007), p. 1179.

[4] J. Qiang et al., in Ref. [3], p. 3522.

[5] M. Venturini, R. Warnock, and A. Zholents, Phys. Rev. ST Accel. Beams 10, 054403 (2007).

[6] M. Venturini, Phys. Rev. ST Accel. Beams 10, 104401 (2007).

[7] S. Heifets, G. Stupakov, and S. Krinsky, Phys. Rev. ST Accel. Beams 5, 064401 (2002).

[8] Z. Huang and K.-J. Kim, Phys. Rev. ST Accel. Beams 5, 074401 (2002).

[9] J. Rosenzweig, C. Pellegrini, L. Serafini, C. Ternieden, and G. Travish, Nucl. Instrum. Methods Phys. Res., Sect. A 393, 376 (1997).

[10] This is actually an impedance per unit length.

[11] J.D. Jackson, Classical Electrodynamics (Wiley, New York, 1974).

[12] W. Fawley, Phys. Rev. ST Accel. Beams 5, 070701 (2002).

[13] I.S. Gradshteyn and I. M. Ryzhik, Table of Integrals, Series, and Products (Academic, New York, 1980).

[14] M. Venturini, Lawrence Berkeley National Laboratory Report No. LBNL-63111, Berkeley, 2007.

[15] A. Chao, Physics of Collective Beam Instabilities in High Energy Accelerators (Wiley, New York, 1993).

[16] C. Bocchetta et al., in Proceedings of the 28th International Free Electron Laser Conference (FEL2006), Berlin, Germany, 2006, available online at http://www.jacow.org.

[17] The laser heater is expected to be normally on during operations.

[18] The spreader directs the beam off the linac axis to the undulators.

[19] M. Venturini and S. Zholents, Modelling Michrobunching from Shot Noise Using Vlasov Solvers, in Proceedings of the Workshop on FEL Frontiers, 2007, Isola d'Elba, Italy [Nucl. Instrum. Methods Phys. Res., Sect. A (to be published)].

[20] D. H. Dowell, in Proceedings of the Workshop on FEL Frontiers, 2007, Isola d'Elba, Italy [Nucl. Instrum. Methods Phys. Res., Sect. A (to be published)].

[21] A. Chao, Z. Huang, and D. Ratner (private communication). 